\title{
Les trames vertes pour les citadins : une appropriation contrastée à Marseille, Paris, Strasbourg
}

Greenways for city dwellers : a mixed ownership in Marseille, Paris, Strasbourg

Sandrine Glatron, Étienne Grésillon et Nathalie Blanc

\section{(2) OpenEdition \\ Journals}

Édition électronique

URL : http://journals.openedition.org/developpementdurable/9297

DOI : 10.4000/developpementdurable.9297

ISSN : $1772-9971$

Éditeur

Association DD\&T

Référence électronique

Sandrine Glatron, Étienne Grésillon et Nathalie Blanc, «Les trames vertes pour les citadins : une appropriation contrastée à Marseille, Paris, Strasbourg », Développement durable et territoires [En ligne], Vol. 3, n² 2 | Juillet 2012, mis en ligne le 12 juillet 2012, consulté le 19 avril 2019. URL : http:// journals.openedition.org/developpementdurable/9297; DOI : 10.4000/developpementdurable.9297

Ce document a été généré automatiquement le 19 avril 2019

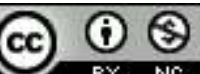

Développement Durable et Territoires est mis à disposition selon les termes de la licence Creative Commons Attribution - Pas d'Utilisation Commerciale 4.0 International. 


\title{
Les trames vertes pour les citadins : une appropriation contrastée à Marseille, Paris, Strasbourg
}

\author{
Greenways for city dwellers : a mixed ownership in Marseille, Paris, Strasbourg
}

Sandrine Glatron, Étienne Grésillon et Nathalie Blanc

1 Interrogés sur ce qu'ils sont susceptibles de formuler concernant la notion et l'application des trames vertes dans leur ville, les citadins demeurent globalement interdits. Ils sont plus diserts lorsqu'il s'agit de parler de nature en ville, ce qui tend à indiquer que les objectifs prêtés à l'aménagement des trames vertes (en premier lieu tournées vers le fonctionnement écosystémique et la préservation de la biodiversité) trouvent un écho, même faible, auprès d'eux. Surtout, s'agissant d'appréhender les dimensions sociales des services écosystémiques que pourraient rendre les trames vertes, il nous est apparu important d'explorer les variations locales de cette politique nationale dont la formulation est, d'abord, nationale et qui trouvera, nécessairement, des déclinaisons locales dans son application. Or les connaissances sur les différences culturelles en matière d'appréciation de la nature sont peu nombreuses. Les travaux de Philippe Descola, anthropologue et auteur de Par delà nature et culture (2005), parmi d'autres, ouvrent le champ d'une réflexion qui vise à agrandir nos imaginations quant à la possibilité d'une pluralité de visions de la nature. Plus modestement, d'autres chercheurs ont montré ces diversités de regard (Kalaora, 1999, Dubost \& Lizet, 2003; numéro thématique de Communications, 2003 pour n'en citer que quelques uns).

2 Nos travaux qui cherchent à caractériser les rapports à la nature et à ces nouveaux outils de l'aménagement que sont les trames vertes, n'ont pas la prétention d'un travail d'une telle portée et ambition. En s'intéressant à trois villes françaises: Paris, Marseille, Strasbourg, ils nous ont permis de distinguer des manières d'appréhender ces sujets sensiblement variables d'un site à l'autre.

3 Nous essayons ici de montrer que les représentations de la nature et des trames vertes en ville permettent de qualifier une esthétique environnementale propre à chaque espace 
urbain. Cette esthétique environnementale s'inscrit dans un corps de pratiques urbaines relevant de la tradition spécifique de la ville concernée. En outre, elle s'accompagne d'une mise en mouvement des différents environnements, d'un «enchantement» de ces derniers (Moscovici (1972) et Marcel Gauchet (1985) qui donne une autre portée à leur analyse. Souscrire à une vision du monde, se représenter l'environnement, mais aussi se doter d'une opinion à son égard, participent, de manière plus générale, d'une activité qui consiste à doter l'environnement d'un effet sur soi ; ainsi, dire de l'herbe qu'elle est sale (papiers, mégots, boue, etc.) ou propre (naturelle, verte, etc.), c'est inscrire dans l'herbe la possibilité de s'y asseoir ou non.

4 La première partie de notre article, en campant les éléments méthodologiques et contextuels de notre étude, permettra de cerner les enjeux de notre questionnement. La deuxième partie, par l'exposé des résultats, permettra de mettre en perspective les représentations et les pratiques de l'environnement urbain déclinées suivant les différentes villes. La troisième partie ébauchera une réflexion sur les rapports qu'entretiennent les citadins à une esthétique environnementale différenciée selon les lieux et les groupes en présence.

\section{Les villes et leurs habitants face aux trames vertes: une enquête par focus groups}

5 L'étude a pris comme point d'appui trois agglomérations françaises très différentes: Paris, Marseille et Strasbourg. Chacune d'elle a des projets d'urbanisme (lotissement, valorisation de ceinture verte, etc.) en cours de réflexion et/ou de réalisation qui intègrent des continuités vertes pour le confort des citadins ou l'émergence de nouvelles formes urbaines plus «nature ». À ces similarités s'opposent des différences liées à l'histoire, à la sociologie et à la géographie de ces agglomérations.

6 Paris (carte 1), capitale nationale, est une ville insérée dans une vaste zone urbaine qui dépasse les 10 millions d'habitants et s'étend sur $2845 \mathrm{~km}^{2}$ (unité urbaine). Le centre ville, très restreint $\left(105 \mathrm{~km}^{2}\right)$, se caractérise par une grande minéralité et une très forte densité humaine et urbaine: avec ses 2,2 millions d'habitants intra-muros, la densité s'élève à 20980 habitants / $\mathrm{km}^{2}$ (chiffres INSEE 2008). Divers projets urbains vont permettre d'expérimenter et d'évaluer l'intégration de la biodiversité dans des continuités paysagères. A titre d'exemple, un périmètre d'étude et de préservation de la biodiversité a été programmé, Paris Nord-Est (19ème arrondissement) avec la matérialisation de grandes continuités écologiques - canaux, voies de chemins de fer, espaces verts... - et les petites parcelles naturelles dont le devenir est à l'étude (jachères fleuries, jardin partagé pour les habitants ou jardin pédagogique pour les enfants des écoles du quartier); une première livraison est prévue en 2012 et la réalisation s'échelonnera jusqu'en 2030. Autre exemple, la promenade plantée sur la Petite ceinture dans le 15ème arrondissement est un projet déjà réalisé sur des emprises ferroviaires avec des associations d'insertion reliant deux grands parcs parisiens : Georges Brassens et André Citroën. Parallèlement, d'ici 2014, un schéma de la trame verte et bleue sera élaboré à Paris et constituera une déclinaison fine du Schéma régional de cohérence écologique (SRCE).

7 Marseille (carte 2), deuxième ville de France, avec ses 851000 habitants, est une métropole insérée dans un écrin «naturel »: la mer Méditerranée à l'ouest, les massifs 
collinéens de l'Estaque, de l'Etoile, de Saint-Cyr et de Marseilleveyre, ainsi que les monts Puget et du Garlaban sur le reste de son pourtour. Bien moins dense que Paris intramuros, avec $3538 \mathrm{hab} / \mathrm{km}^{2}$, la commune est particulièrement étendue : avec $240 \mathrm{~km}^{2}$, c'est l'une des plus grandes communes de France métropolitaine, d'où sa faible densité. En effet, elle intègre les espaces à forte naturalité que sont les calanques et les collines sus-citées. Une description des espaces verts et des éléments d'une infrastructure "verte» affichée par les services de planification figure dans l'article de Consales et al. (2012 - dans le même dossier que cet article).

Strasbourg (carte 3), enfin, accueille le tiers (272 000) des 758000 habitants de l'aire urbaine (chiffres et nomenclature INSEE 2008) sur un territoire communal de $78 \mathrm{~km}^{2}$, soit une densité urbaine équivalente à celle de Marseille avec 3477 habitants $/ \mathrm{km}^{2}$. Le projet de trame verte est seulement en cours de montage au sein de la mairie et de la communauté urbaine (CUS) ; mais 24 projets s'inscrivent depuis 3 ans maintenant dans la démarche EcoCité lancée dans le cadre du plan ville durable présenté par Jean-Louis Borloo en octobre 2008 (13 territoires, parmi lesquels Strasbourg, ont été sélectionnés en 2009). Ainsi, la « capitale européenne » qui trouve son prolongement naturel outre-Rhin avec la ville de Kehl, peut-elle s'enorgueillir de contribuer à construire un territoire urbain durable, même si la trame verte, telle que définie dans le Grenelle de l'environnement est encore au stade de la réflexion. On peut ainsi souligner que les continuités, qui émergeront officiellement dans le cadre de cette trame verte et bleue urbaine, sont, d'ores et déjà, émaillées de projets urbains en cours de réalisation ou planifiés à court terme.

Carte 1. La ville de Paris et son maillage d'espaces verts

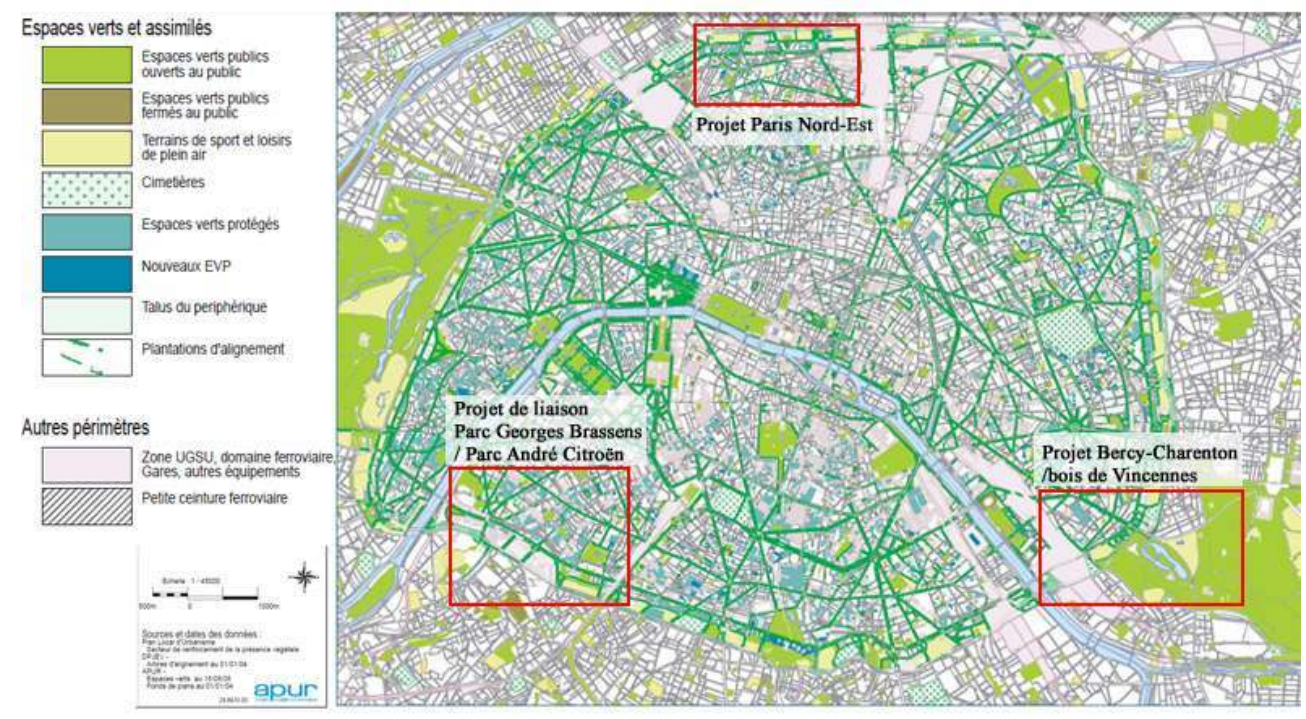

Source : APUR, 2005 : 5 
Carte 2. Marseille en négatif

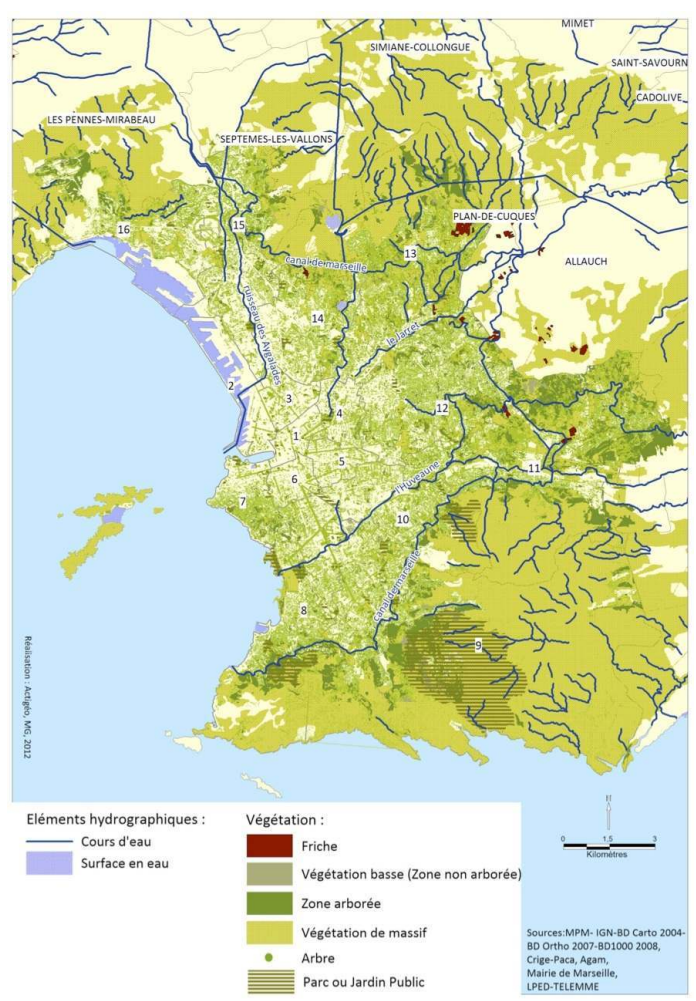

Source : Consales et al., http://developpementdurable.revues.org/9268

Carte 3.a ville de Strasbourg et son maillage d'espaces verts

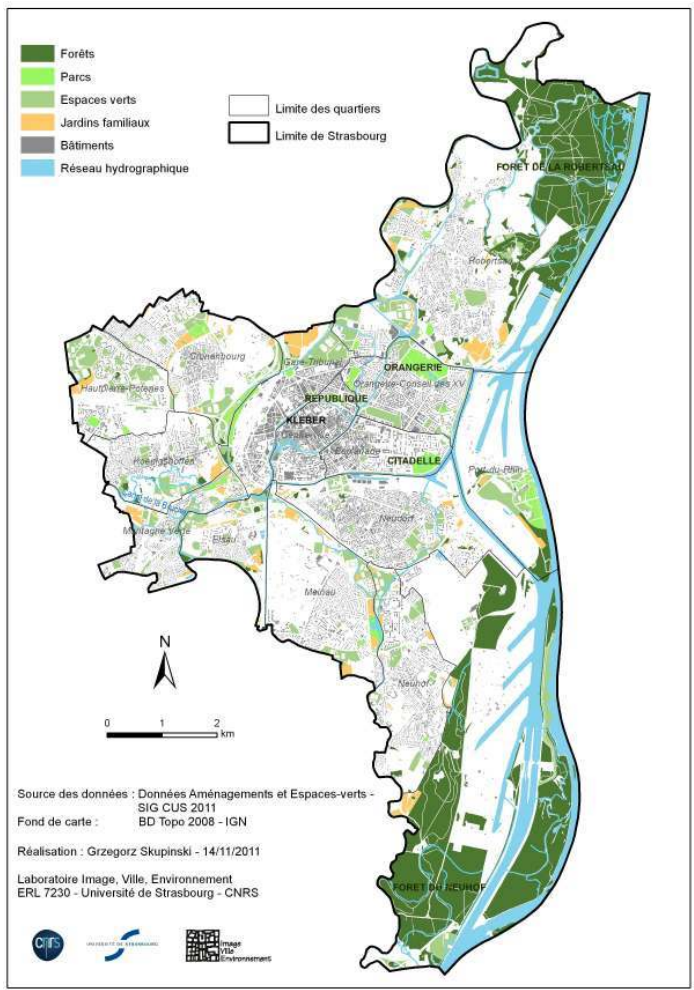

Source : LIVE 2012 
Les habitants de ces trois villes vivent dans des contextes urbains très variés ce qui nous paraissait intéressant pour vérifier de quelle manière leur environnement, au sens géographique, politique et culturel, influence leurs représentations et pratiques des trames vertes qui, elles, découlent d'une politique urbaine nationale. Nous avons cherché à constituer des groupes susceptibles d'être représentatifs de la population urbaine par les catégories sociales, les caractéristiques démographiques et professionnelles. En outre, nous avons posé comme hypothèse que le genre des individus, l'aspect du paysage urbain fréquenté quotidiennement ainsi que la sensibilité politique aux questions environnementales peuvent avoir une incidence sur les pratiques et représentations de la ville, de la nature et, partant, de la trame verte. Aussi, nous avons panaché 3 critères pour constituer les groupes: le genre (hommes vs femmes), la localisation de l'adresse de résidence (centre-ville vs quartiers périphériques) et la «sensibilité environnementaliste " (non sensible a priori vs militants écologistes ou votants verts) (tableau 1). Le panachage a également inclus un critère de professions et catégories socioprofessionnelles (CSP) en nous référant à la nomenclature INSEE de niveau 1 ' 8 postes) : nous avons cherché une représentativité socio-professionnelle à l'échelle de l'ensemble des enquêtés, pour chaque ville.

$\mathrm{Au}$ total, nous avons conduit huit focus group par ville, chacun composé de six à neuf personnes réunies autour de deux à trois chercheurs, soit un total de vingt-quatre groupes, permettant de rencontrer plus de 160 citadins. Nous ne pouvons certifier que la méthode permet de faire émerger la diversité des représentations mais le nombre non négligeable de citadins, les différents critères socio-spatiaux de sélection décrits cidessus, ainsi que certaines redondances dans les propos nous incitent à penser qu'à défaut de représentativité, nous sommes face à une significativité satisfaisante des propos.

11 La technique des focus group, entretiens collectifs où l'animateur/modérateur formule quelques questions (six dans notre cas) et laisse s'exprimer, se répondre, se contredire les personnes interrogées, s'inspire des résultats des travaux de dynamique de groupe développés par la psychologie sociale (Mucchielli, 2004 ; Catterall \& Maclaran, 1997). Cette technique nous a paru intéressante pour aborder un sujet de société : même si les conditions en sont limitées (temps, espace, choix restreint de personnes), le groupe de parole permet de proposer un espace de débat qui, par certains côtés, se rapproche du débat public qui nous semble devoir être à l'oeuvre dans la réflexion sur les trames vertes.

Tableau 1. Profils des huit focus groups des trois agglomérations

\begin{tabular}{|l|l|l|l|}
\hline Profil groupe & Femmes & Hommes & Mixte \\
\hline Centre & 1 & 1 & $\begin{array}{l}1 \text { (militant écologiste) } \\
1 \text { (non militant) }\end{array}$ \\
\hline Périphérie & 1 & 1 & $\begin{array}{l}1 \text { (militant écologiste) } \\
\text { (non militant) }\end{array}$ \\
\hline
\end{tabular}




\section{Représentations et pratiques de l'environnement urbain : des villes, des natures, des trames vertes}

\subsection{Analyse de contenu : des différenciations marquées}

Après retranscription de la totalité des enregistrements, l'étude du corpus s'est appuyée sur une approche lexicométrique et une analyse de contenu. Les analyses lexicométriques ont souvent mis au jour des hypothèses que l'analyse de contenu a permis de vérifier ou d'infirmer. Malgré une simplification de l'énoncé, les analyses lexicométriques offrent à un regard averti un outil intéressant pour délimiter des formes textuelles originales, pour comparer des corpus textuels (Lafon, 1981). Pour ce travail, nous avons utilisé deux logiciels de lexicométrie: Lexico3 (réalisé par l'équipe universitaire SYLED-CLA2T, Université de Paris 3, et distribué par ILPGA, Paris) et Alceste (édité par la société Image, Toulouse).

13 Lexico permet de dénombrer les occurrences de mot: anecdotique sur des mots très courants ce dénombrement peut s'avérer signifiant sur des mots plus rares. Le croisement de l'occurrence des mots avec le profil du focus group et le site de l'entretien donne un éclairage nouveau sur les sites d'études et les personnes interrogées. Lexico identifie également des co-occurrences de mots qui permettent de comprendre les liens effectués entre différents termes par les personnes interrogées.

Avec le second logiciel, Alceste, le texte est intégré à l'échelle d'associations regroupant quelques mots, appelées Unités de Contexte Elémentaires (UCE). À partir d'une classification ascendante hiérarchique, les distances entre tous les mots du corpus textuel des focus group sont calculées et des UCE générées. Ensuite, il regroupe les UCE en classe qui rassemble des segments de discours de plusieurs paragraphes. Ces classes peuvent ensuite être associées avec les profils des groupes, avec les personnes interrogées, ou avec les sites d'enquête.

L'analyse de contenu a permis de classer et d'organiser comme éléments de communication les éléments des discours adressés par les personnes au groupe. Des portions de contenus des focus group, en réponse aux questions, ont été isolées et ont fait l'objet d'une lecture attentive. Les réponses ont été segmentées suivant une catégorisation correspondant aux questions. Par exemple, à la question "Quelles pratiques de la nature avez-vous?", les réponses décrivant des actions, (se promener, jouer, observer, etc.) ont été mises à part et décrites comme des entrées; ainsi, les différentes actions correspondantes à l'idée de nature ont été restituées et interprétées par ville. Nous avons privilégié les comparaisons entre villes, aux dépens des critères de différenciation entre groupes (femmes/hommes, environnementalistes / nonenvironnementalistes) qui ne se sont pas montrées pertinentes, sur le plan lexicométrique. La différence centre/périphérie semble comporter plus d'informations.

Sur un corpus réunissant les discours des habitants des trois villes (421 210 formes), la classification descendante effectuée à partir d'unité de texte d'Alceste a établi huit classes différentes (tableaux 2 et 3 ). 
Tableau 2. Classes regroupant les mots les plus utilisés $(\Sigma)$ et les plus significatifs $\left(\Sigma K \mathrm{KI}^{2}\right)$ : l'importance de la ville d'appartenance.

\begin{tabular}{|l|l|l|l|l|l|l|l|l|l|l|l|}
\hline \multicolumn{2}{|l|}{ Classe 1 } & \multicolumn{4}{|c|}{ Classe 2 } & \multicolumn{3}{c|}{ Classe 3 } & \multicolumn{3}{c|}{ Classe 4 } \\
\hline $\begin{array}{l}\text { Mot/ } \\
\text { descripteur }\end{array}$ & $\Sigma$ & $\sum \mathrm{KHI}^{2}$ & $\begin{array}{l}\text { Mot/ } \\
\text { descripteur }\end{array}$ & $\Sigma$ & $\sum \mathrm{KHI}^{2}$ & $\begin{array}{l}\text { Mot/ } \\
\text { descripteur }\end{array}$ & $\Sigma$ & $\sum \mathrm{KHI}^{2}$ & $\begin{array}{l}\text { Mot/ } \\
\text { descripteur }\end{array}$ & $\sum$ & $\begin{array}{l}\sum \mathrm{KHI} \\
2\end{array}$ \\
\hline Paris & 376 & 517 & Marseille & 521 & 456 & Espèce & 116 & 402 & Politique & $\begin{array}{l}15 \\
5\end{array}$ & 380 \\
\hline Pigeon & 88 & 402 & Poubelle & 248 & 325 & Animal & 112 & 288 & Ecologique & $\begin{array}{l}27 \\
1\end{array}$ & 329 \\
\hline Rat & 80 & 240 & Ramasser & 46 & 163 & Végétal & 52 & 280 & Grenelle & 51 & 157 \\
\hline Ecureuil & 40 & 225 & Chien & 80 & 147 & Corridor & 56 & 143 & Recherche & 48 & 104 \\
\hline Poisson & 30 & 131 & Merde & 20 & 68 & $\begin{array}{l}\text { Strasbourg } \\
(*)\end{array}$ & 183 & 85 & $\begin{array}{l}\text { Dévelop- } \\
\text { pement }\end{array}$ & 28 & 80 \\
\hline
\end{tabular}

Tableau 3 : Classes regroupant les mots les plus utilisés $(\Sigma)$ et les plus significatifs $\left(\sum K \mathrm{KI}^{2}\right)$.

\begin{tabular}{|c|c|c|c|c|c|c|c|c|c|c|c|}
\hline \multicolumn{3}{|l|}{ Classe 5} & \multicolumn{3}{|l|}{ Classe 6} & \multicolumn{3}{|l|}{ Classe 7} & \multicolumn{3}{|l|}{ Classe 8} \\
\hline $\begin{array}{l}\text { Mot/ } \\
\text { descripteur }\end{array}$ & $\Sigma$ & $\sum \mathrm{KHI}^{2}$ & $\begin{array}{l}\text { Mot/ } \\
\text { descripteur }\end{array}$ & $\Sigma$ & $\sum \mathrm{KHI}^{2}$ & $\begin{array}{l}\text { Mot/ } \\
\text { descripteur }\end{array}$ & $\Sigma$ & $\sum \mathrm{KHI}^{2}$ & $\begin{array}{l}\text { Mot/ } \\
\text { descripteur }\end{array}$ & $\Sigma$ & $\sum \mathrm{KHI}^{2}$ \\
\hline Voiture & 324 & 1030 & Trame & 463 & 1342 & Habiter & 280 & $\begin{array}{ll}917 \\
\end{array}$ & Parc & 406 & \begin{tabular}{|l|}
257 \\
\end{tabular} \\
\hline Transport & 105 & 576 & vert (e) & 459 & 827 & Appartement & 105 & 665 & Arbre & 281 & 164 \\
\hline Vélo & 106 & 251 & Continuité & 174 & 476 & Vécu & 48 & 346 & Fleur & 118 & 161 \\
\hline Parking & 56 & 233 & Evoquer & 108 & 389 & Maison & 83 & 267 & Joli & 67 & 112 \\
\hline Metro & 63 & 197 & Représenter & 35 & 106 & Banlieue & 49 & 246 & Sauvage & 108 & 100 \\
\hline
\end{tabular}

Le logiciel n'opère pas les distinctions à partir des descripteurs et des différents locuteurs mais il effectue des classes en fonction de moments, dans le discours, pendant lesquels le champ lexical est homogène. Ainsi, la classification montre que les habitants ont, à certains moments de l'entretien, des discours spécifiques en fonction de leur ville d'appartenance. Ainsi, la classe 1 délimite principalement les dialogues des Parisiens, la classe 2 repose essentiellement sur les échanges marseillais et les discours strasbourgeois s'associent en grande partie dans la classe 3 (tableau 2). A ces différences géographiques s'ajoute une classe propre aux discours des chercheurs animant le débat (classe 4, tableau 2). Nous retrouvons les mots employés dans l'introduction ou dans la conclusion (politique, recherche, Grenelle) et dans les questions (écologique).

18 Une classe regroupe les réponses à la première question où les participants étaient invités à présenter leurs lieux de vie (classe 7, tableau 3). Ces parties de discours rassemblent les termes propres à la description de l'habitat (maison, appartement) et du quartier (banlieue, centre).

19 Enfin 2, 6 et 8 (tableau 3) classes formalisent deux manières de présenter la nature. L'une se rapporte à l'individu (classe 6), l'autre est une nature décrite de manière extérieure à l'être humain (classe 8 , tableau 3). De manière plus sectorielle, en fonction des villes considérées, nous observons des différences de groupes relativement marquées.

\subsection{Strasbourgeois environnementalistes : un discours très typé, des continuités naturelles structurantes}

20 Afin de ne pas induire trop rapidement une réponse attendue de nos citadins, nous avons pris soin de commencer les entretiens par un questionnement général sur le choix de leur lieu de vie, puis sur la nature. Interrogés sur ce qu'est pour eux la nature dans leur ville et où on la trouve, les citadins de nos trois villes émettent des propos qui se ressemblent 
beaucoup à l'exception des Strasbourgeois environnementalistes qui constituent un ensemble à part, tout à fait singulier, où les thématiques centrales des trames vertes, comme aménagement destiné en premier lieu à préserver la biodiversité, sont abordées spontanément et très rapidement. Pour les autres, environnementalistes ou non, femmes ou hommes, Parisiens ou Marseillais sont assez démunis quant au concept de trame verte ou à son application (voire son applicabilité) dans leur ville.

Le premier constat, frappant, est la mention explicite de la trame verte par les groupes environnementalistes strasbourgeois très rapidement au cours des entretiens, alors que ni le vocable ni même la notion n'ont encore été évoqués par les animateurs et qu'il s'agit seulement de répondre à la première question : " qu'est-ce que la nature pour vous ? Où la voyez-vous?». Ainsi, évoquant l'habitat nécessaire à la présence des loriots dans la ville, deux hommes, habitant les quartiers périphériques échangent :

"- On ne va pas trouver un loriot place Kleber: même s'il y a quelques arbres, ça ne le fera pas, quoi. Et puis il y a de l'eau pas loin.

- Il faut une certaine continuité de verdure. Une trame, en fait, une trame verte. »

Ou encore, à la sème minute de l'entretien :

F1 (environnementaliste, quartiers péricentraux): "Enfin moi, quand j'entends nature en ville, c'est ça que ça m'évoque. C'est de me dire: Ah tiens, maintenant, il y a les arbres qui poussent entre le goudron, ça pousse le goudron, il y a des petites fleurs qui poussent entre les galets... plus que tous les territoires verts qu'il y a vers Koenigshofen, Montagne Verte, Elsau, en fait toute la trame verte, quoi. »

F2 (Strasbourg ville, quartiers péricentraux, environnementaliste) : pour moi, continuité écologique, c'est trame verte, quoi !

F3 (Strasbourg ville, quartiers centraux, environnementaliste) : ben si, on connaît. Ce sont des plans (...) : ce sont des circuits piétons et cyclistes le long de parcs et de... oui, de régions fleuries et boisées, le long des jardins. Vous ne connaissez pas ces circuits « trame verte »?"

Même quand le terme n'est pas explicitement cité, on trouve, dans la description des lieux de naturalité, cette idée forte de la continuité permettant le cheminement des espèces que les trames vertes sont destinées à favoriser :

H1 (environnementaliste, quartiers centraux): "en tout cas, ce sont des petits bouts de nature ; ça sert aussi de passerelles, les petits bouts de nature. Voilà oui, pour les oiseaux, papillons, c'est des passages, des passerelles, comme ça. "

H2 (environnementaliste, quartiers centraux) : « oui, oui, oui. Bon, le long du canal de la Bruche également (...). C'est une sorte de traînée verte qui est très agréable. »

\subsection{Citadinité et trame verte : la circulation des hommes en premier lieu}

Pour les citadins des autres villes, le terme «trame verte » renvoie essentiellement au "tram" (nouvelle politique publique en matière de déplacement avec des effets structurants sur les espaces urbains), à la coulée verte évoquée par les Parisiens et les Marseillais. Aux questions successives concernant les termes de continuité naturelle, 
corridor écologique et trame verte, les participants aux focus groups ont des réponses qui témoignent de leur capacité à penser ces objets dans les espaces qu'ils habitent. Si les Strasbourgeois sont clairement ceux qui évoquent le plus naturellement ces espaces qualifiés de linéaires allant de l'extérieur des villes vers les centres, ce sont a contrario les Parisiens qui ont le plus de mal à imaginer ce que pourraient signifier de tels aménagements dans un espace considéré comme exceptionnel. Par exemple, se baigner dans la Seine à Paris ou en aval n'est pas se baigner dans la même eau, tant le contexte joue dans la qualification des espaces. Les continuités sont, dès lors, difficiles à imaginer. Pour les Marseillais la trame verte renvoie, d'une part, à leur aménagement récent du tramway, qui est un échec selon eux, et, d'autre part, à des possibilités ouvertes à partir des collines de relier des linéarités.

Les trois expressions renvoient bien évidemment par effet d'approximations successives à partir du sens des mots (naturel, continuités, corridors, trame) à des significations bien différenciées. La continuité naturelle comporte une dimension temporelle, liée à la nature, associée à l'évocation de la continuité dans un contexte de déploiement des politiques de développement durable. Le corridor écologique évoque la préservation, l'enfermement associé au mot corridor, ou couloir. Les trames vertes sont associées (mais pas spontanément, loin s'en faut) à un aménagement maillant la ville, resituant la ville dans la nature ; pour les Strasbourgeois rencontrés, la trame est visible au quotidien :

F (Strasbourg, quartiers péri-centraux, environnementaliste) : "A Strasbourg, là, pour le coup, il faut séparer le concept global « ville » et Strasbourg. Parce qu'il y a quand même des efforts importants, en particulier sur toutes les voies cyclables, etc., qui sont quand même globalement vertes, quoi. Toutes les pistes cyclables sont des espaces de respiration à Strasbourg. Et on a la chance d'en avoir beaucoup"

Les éléments d'une trame verte apparaissent physiquement plus présents à Strasbourg, en particulier parce que les citadins les investissent comme l'espace d'une pratique, la mobilité quotidienne. Celle-ci induit, presque par essence, des continuités qui sont donc perceptibles, perçues et décrites.

À Paris, un homme habitant Saint-Ouen antérieurement le dit, en même temps que son scepticisme sur la possibilité d'une trame verte :

"Si on me dit trame verte j'imagine un maillage, peut-être quelque chose qui ferait le lien entre la ville et la campagne, mais c'est vrai que je n'arrive pas à le visualiser, je ne vois pas quelle forme ça pourrait prendre sur une grande ville comme Paris, surtout c'est vrai qu'on a... il y a le périphérique qui est là, je ne vois pas quelles sont les portes d'entrée pour la nature dans Paris, j'imagine que l'idée de trame c'est aussi qu'on l'aide un petit peu à venir jusqu'à nous, mais je... je n'arrive pas à l'imaginer... On pourrait penser à des réalisations dans chaque arrondissement..."

28 À Paris et Marseille, les éléments de nature sont plutôt liés à des pratiques stationnaires, dans les parcs :

F (Marseille centre, non environnementaliste) "moi le jardin c'est statique, c'est la bronzette, le livre, c'est à tout casser $1 \mathrm{~h} 30$, et je vais vous expliquer pourquoi : c'est très simple, parce qu'il y a des chiens." 
Dors, la continuité est nettement moins facile à projeter ou à percevoir. Marseille présente un cas de figure singulier, bien différent des autres avec la présence de calanques sur le territoire communal, à proximité du centre dense : le lien avec ces espaces de nature, très marquants et présents dans tous les esprits, est rendu ambigu par cette inclusion (administrative, bien connue) - extériorité (il s'agit vraiment de réserves naturelles).

\subsection{Des pratiques environnementales très typées selon les villes}

Ainsi, les pratiques autour de la nature et des éléments de trames vertes diffèrent profondément selon que l'on se trouve à Paris, Marseille ou Strasbourg. Elles sont liées aux pratiques et représentations des milieux concernés (parcs urbains, bords de mer ou de fleuve), et, pour les trames vertes, aux continuités existantes.

De manière globale, les habitants de Strasbourg observent et contemplent la nature en marchant, à vélo ou se rendent à l'extérieur de la ville. Les motifs esthétiques, le plaisir de l'observation de la vie animale sont évoqués à de nombreuses reprises par nos interlocuteurs. Une des femmes explique ses pratiques de nature :

F (Strasbourg, quartier central, environnementaliste) "La reconnaissance des espèces, l'observation. Quand ça se présente, l'éducation aux enfants, qui adorent les connaître, nommer. Il y a une poésie aussi. (...) C'est assez dingue parce qu'il y a beaucoup d'enfants qui sont sensibilisés, et qui aiment savoir : les noms. Voir la beauté. Ca marche bien."

La transmission et l'éducation figurent au registre des actions réalisées autour de la nature, mêlant cognition et émotion : il a été souligné dans d'autres travaux et dans la littérature combien la connaissance peut modifier le regard sur la nature : «l'aspect cognitif participe pleinement de notre évaluation esthétique » (Luxerau, 2003).

Enfin, la nature représente une nécessité et s'y ressourcer un objectif majeur de la vie citadine comme en témoignent ces propos :

F (Strasbourg, quartier central, environnementaliste) "Je vis la nature à deux titres: d'une part, en termes d'observation. Observer cet espace-là ; et d'autre part, essayer d'en faire rentrer davantage. D'abord, pour des questions de santé, c'est sûr. Pour des questions de nécessité vitale ; ce n'est pas simplement une nécessité vitale parce que ça produit tout un tas d'éléments vitaux pour notre corps, mais plutôt parce que c'est une nécessité vitale d'une manière psychologique. Je pense qu'un homme, un être humain entouré de béton, il finit par devenir de la pierre, quoi. Qu'il lui faut un peu de vie, quelque chose qui bouge, quelque chose qui le rapproche de ce qu'il est lui, graine, terre, voilà... et que... cet effet d'observer la nature, de la contempler, de la faire revenir, de le faire de manière quasiment militante, c'est-à-dire en parvenant à joindre dans cette observation d'autres personnes, ça permet de créer un tissu urbain qui, lui, se remet à vivre et qui permet d'accéder à une humanité autre".

La nature renvoie à une continuité spatiale, celle d'un cadre de vie, notre sol, mais aussi d'une continuité intergénérationnelle. En somme, comme le résume très bien une autre femme, habitant le Neudorf, divisant son environnement en cercles successifs et, donc, non plus suivant des lignes de fuite (chemins, canaux, etc.) : 
F (Strasbourg, quartier péri-central, non environnementaliste) "Je vis avec la nature, sinon je ne vis plus. Dans l'appartement où je suis, je peux profiter d'un jardin derrière, d'un potager (...). Là, c'est un espace où on peut se mettre dehors, bouquiner au calme (...). C'est la première utilisation que je fais de la nature. Ensuite, ce sont les parcs, si je dois me dépenser avec mon fils, jouer au foot ou n'importe quoi. Après il $\mathrm{y}$ a les parcs où j'aime profiter si j'ai un petit moment seule. Ça peut aussi m'aider d'entendre qu'il y a du monde autour. Ce sont des lieux où je me sens en pleine nature pour bouquiner ou autre. Et puis, ensuite, il y a la pleine nature où on essaie d'aller le plus souvent (mais on ne peut pas non plus partir tous les quatre moments). Je fais un peu d'escalade en plein air. Donc là, on est en pleine nature : montagne, randonnée... “

Les Marseillais décrivent une fréquentation dédoublée de la nature : d'un côté les parcs (Borely, Longchamp, etc.) qui composent la nature urbaine où se retrouvent de nombreux problèmes de civilités; de l'autre, les calanques qui représentent des espaces plus authentiques mais ressentis comme extérieur à l'urbanité et à ses règles et désordres.

H (Marseille centre, non environnementaliste) : "il y a le parc Borely, il y a le parc du 26ème millénaire, il y a le parc Longchamp... pour ne parler que par ici, ensuite il y en a d'autres, il y a de beaux parcs, le parc Berger tout ça... (...) mais malheureusement j'ai constaté avec les Marseillais que, depuis quelques années, il y a de moins en moins de civisme, les gens ne respectent plus ça, c'est un peu dommage..."

Les calanques et les lieux extérieurs à la ville sont évoqués systématiquement comme des refuges ou des espaces d'évasion. L'espace urbain est antinomique d'un espace naturel. Deux femmes non-environnementalistes habitant le Marseille périurbain l'expliquent, chacune à sa manière :

F (Marseille péricentre, non environnementaliste) :"Si je veux promener les enfants, honnêtement, je préfère aller en bord de mer dans les calanques sauvages, au moins je suis sure qu'elle n'a pas été abîmée: il n'y a pas de constructions, c'est naturel, c'est sauvage, et puis vraiment on voit la nature, on voit ses animaux, ses bestioles, et puis on voit de la verdure, on voit la nature sauvage..."

F (Marseille péricentre, non environnementaliste) “Je m'éloigne, je vais sur les calanques, je crapahute avec mon gamin et mon chien, donc je vais carrément du côté des Goudes, mais j'ai vraiment moi ce sentiment d'étouffer, dans mon secteur j'étouffe, et si je peux éviter un maximum de gens je les évite, parce que de toute façon ça n'apporte rien de plus dans le sens où c'est que du brassage, c'est que... j'ai vraiment besoin moi pour me « ressourcer » entre guillemets, d'avoir un environnement qui m'apaise, soit par la vue, par le soleil qui est à l'horizon, la mer, d'entendre ces animaux, ces guêpes, de voir ces petits moucherons, de voir ces petites fleurs..."

37 À Paris, les parcs et jardins sont systématiquement évoqués tandis que la Seine ne figure jamais au titre de nature. Les gens viennent s'asseoir, lire, se reposer, écouter de la musique, faire jouer leurs enfants et, souvent, courir dans ces parcs. Quelques forêts périurbaines jouent un rôle dans le déploiement de pratiques de nature : Fontainebleau, Rambouillet, etc.

$\mathrm{Au}$ total, les Strasbourgeois évoquent une nature qui renvoie à leur mobilité; tandis que les Parisiens et les Marseillais se rendent au parc et s'y déplacent peu. La mobilité est 
souvent réservée aux espaces urbains et consiste à relier un point à un autre. Le parc, l'endroit de nature, sont des espaces en rupture avec la mobilité frénétique urbaine. Les citadins y viennent pour trouver, le repos, le calme (opposé au stress, au bruit, à l'agitation des citadins ou de la circulation), la tranquillité. La nature est également associée à une certaine pureté (opposée à la pollution en particulier liée à la circulation) :

F (Strasbourg, centre-ville, non-environnementaliste) : «mais même pour me promener avec mon chien. C'est une façon de décompresser. On s'oxygène, ça fait du bien ».

Elle parle aux sens, aux sentiments :

$\mathrm{H}$ (Strasbourg, quartiers péri-centraux, non-environnementaliste) : « $\mathrm{Au}$ niveau des odeurs, c'est aussi beaucoup plus sympa. Et visuellement, c'est aussi un gros facteur au niveau des saisons, inconsciemment (les feuilles qui sont sur le sol)».

$\mathrm{H}$ (Strasbourg, centre-ville, environnementaliste) : " par tous les sens, on va dire. Là, en ce moment, vous allez dans certains coins, ça sent l'accacia à plein nez. Vous avez un autre coin, c'est du sureau. C'est... Fffff. Même l'odeur de la pluie sur l'herbe, c'est... pour moi, c'est toutes les sensations. Ça peut être le toucher... voilà ».

F (Paris, centre-ville, environnementaliste) «la nature, c'est reposant, donc le vert c'est reposant. Parce qu'on n'est pas dans l'agitation de la ville ».

Finalement, l'espace vert fréquenté renvoie essentiellement aux sensorialités (Bridge, 2008). La nature, pour certains, a quelque chose de synthétique et ne paraît pas tout à fait " vraie » lorsqu'elle est en ville. Cependant, elle est, essentiellement, pourvoyeuse d'un bien-être dans lequel les sens ont un rôle prépondérant. Jusqu'au climat, mentionné comme un élément important dans le bien-être des citadins, associé à la nature. Ces remarques jouent un rôle important pour comprendre le rôle éventuel des trames vertes dans l'espace urbain. Une esthétique environnementale, champ de recherches d'origine anglo-saxonne portant sur l'engagement esthétique dans l'environnement, peut permettre de développer ce rôle et d'enrichir la compréhension de ces nouveaux aménagements. En effet, les trames vertes vont modifier les rapports à la nature en ville et celui des villes à leur environnement naturel local et régional. Elles pourraient également contribuer à une meilleure adaptation des systèmes urbains au changement climatique. L'écologie du paysage et la géographie sont à l'origine de cette conception du territoire et de son aménagement (Arrif T., Blanc N., Clergeau P., 2011). Cependant, ce terme d'usage est restreint à une sphère d'experts et de politiques; donc il importe donc de comprendre la place donnée à la nature, au-delà de celle des trames vertes, et comment les trames vertes s'y relient.

\section{Le face à face citadins / nature : une esthétique de l'environnement}

\subsection{Sensorialités et investissement actif}

Quels enseignements tirer de cette lecture des focus groups, en termes d'esthétique environnementale? Rappelons que l'esthétique environnementale, courant essentiellement anglophone, inspiré des philosophies de la nature, insiste sur 
l'expérience esthétique pour les environnements naturels. Selon les théoriciens de ce courant, deux approches principales existent : les approches cognitives et les approches non cognitives ou « émotives » (Brady, 2003).

Pour des auteurs tels Allen Carlson (1999, 2004) ou Holmes Rolston (2007), la connaissance, en particulier scientifique (écologie, géologie, etc.) guide nos appréciations de la nature (Blanc, 2008). L'accent est placé sur les dimensions d'objectivité, de réalité et de vérité des mondes dépeints. La deuxième catégorie d'auteurs, parmi lesquels Arnold Berleant (1992 ; 2010), Yuriko Saito (2010), Emily Brady (2003; 2007), se fonde sur l'expérience perceptuelle immédiate et profonde ; l'imagination, l'émotion, le plaisir, les récits non scientifiques, l'oralité étant considérés comme des éléments constitutifs de l'expérience esthétique où le sujet trouve sa place. Cela inclut folklore, mythes, récits vernaculaires, croyances. Les dimensions narratives, métaphysiques et spéculatives de l'être humain dans la nature sont valorisées. L'expérience esthétique est située, contextuelle, jamais abstraite. Elle témoigne de l'effacement de la séparation nature/ culture. Les aspects multi-sensoriels manifestes d'une immersion environnementale sont privilégiés. Cette dernière approche environnementale au sein de laquelle nous inscrivons nos travaux (Blanc N., 2008, 2012 ; Blanc \& Ramos, 2010) permet de décrire les modes d'imagination et de recréation des environnements ordinaires ou exceptionnels. En ce sens, plus que la rationalité scientifique et ses valeurs, l'imaginaire, le symbolique, le perceptuel, le sensoriel sont dominants dans le débat public associé à l'environnement.

Les focus groups mettent en évidence des images globalisantes de l'environnement associées, d'ailleurs, à une éthique. L'esthétique des trames vertes renvoie aux dimensions perceptives, sensorielles des environnements naturels ou construits. Enfin, les qualités attribuées aux environnements verts en ville relèvent, pour l'essentiel, d'une compréhension esthétique des enjeux écologiques. La biodiversité est appréciée en termes de sensorialités : l'abeille et l'oiseau émettent des sons agréables, les odeurs des plantes promettent du bonheur, etc. Dans cette approche de l'environnement, les êtres humains se représentent la nature dans un système changeant, en fonction de la ville, mais également en fonction de critères éthiques qui expriment des sensibilités différentes aux vivants.

Voyons plus spécifiquement les figures ou images spatiales auxquelles renvoient les trames vertes : premièrement, l'idée de trame verte renvoie à ce qui relie le citadin à la nature. Elle met en lumière les liens spatiaux et temporels entre les êtres humains et les autres vivants. Elle renvoie fortement à l'acceptation de la nature par les habitants, qui la réinventent par rapport à leurs représentations. Elle suppose une ouverture des habitants à une nature inconnue qui est qualifiée en fonction des individus de "sauvage ", de " délaissée », de "libre », ou encore de "spontanée ». Pour cette raison, la survie de ces entités repose sur une cause éthique détachée dans un premier temps du destin de l'humanité. Il faut sauver la faune et la flore pour elle-même. L'esthétique de la nature est gratuite, et c'est cette gratuité qui fait sa grandeur. La nature n'est pas présente pour l'humanité, elle est consubstantielle au monde: les trames vertes "c'est la nature qui reprend son droit » (divers enquêtés de toutes les villes). La trame verte est une hétérotopie au sens donné par Michel Foucault (2009): ces espaces constituent des «contreemplacements» par rapport aux mondes anthropisés. Les trames vertes et les hétérotopies délimitent un monde sans expérience anthropique directe. 


\section{2. Écologie et esthétique des trames vertes} l'environnement naturel et construit. Le jugement de goût se construit dans un va-etvient entre social, culture et capacité d'émettre une opinion personnelle. Il joue sur les formes cristallisées de la vie de la Cité de façon à promouvoir des modèles ou des alternatives au sens des visions. Il s'agit bien d'émotion et de mettre en avant ce qui ne peut l'être sans bousculer les conventions, normes et règles d'un groupe social; une certaine créativité, la vision d'un changement, d'un déplacement rendu nécessaire, une beauté à l'œuvre encore invisible. Pour les trames vertes, nous partageons les résultats décrits par Paul Gobster, Joan I. Nassauer, Terry C. Daniel et Gary Fry (2007) : ils mettent en évidence l'importance du contexte dans l'expérience esthétique. Il existe un décalage entre les appréciations esthétiques et écologiques de la nature. Un paysage ou un coin de nature apprécié n'est pas forcément écologiquement raisonnable. À la différence des travaux susnommés, nous ne voulons pas ajuster les appréciations esthétiques sur le raisonnement écologique. Une telle approche serait normative; or, nous avons essayé d'être compréhensifs et descriptifs. Que faire, donc, de ce décalage? Constatons tout d'abord, et les propos des Strasbourgeois l'expriment, que les valeurs paysagères évoquées donnent une plus grande place au sauvage, au naturel, à la biodiversité. Lentement, certes, au regard des enjeux considérés, les appréciations esthétiques se transforment en fonction de nouvelles normes écologiques. Les gens ne se rallient pas aux valeurs écologiques per se, mais leur positionnement social répond à ces valeurs (notamment anti-productivistes). Notons également, à l'issue de ces travaux, que si les représentations et pratiques humaines jouent sur la production de l'environnement, il est remarquable qu'à l'inverse, l'environnement, le site affecte les perceptions, renvoie à du matériel interagissant avec les organismes, du symbolique (le vert comme rappel du Paradis), de l'imaginaire (la ville du futur est là), de l'affectif ( « c'est sur cette pelouse que je l'ai rencontré »), de l'évaluatif (« ce beau paysage vert »). La complexité des processus environnement-humains montre que les connaissances, les savoirs de tous ordres jouent un rôle dans l'évolution des perceptions.

\section{Conclusion}

Parce que nous avons voulu savoir quelles significations les citadins attribuent aux trames vertes urbaines, aménagement « phare » du Grenelle de l'environnement destiné à lutter contre l'érosion de la biodiversité, nous avons interrogé les habitants de trois grandes villes françaises. La perception de ces trames vertes urbaines, nécessairement déclinées à l'échelle locale, fait écho à la diversité des contextes, des politiques et des environnements urbains ainsi que des cultures locales. Dans tous les cas, l'importance des sensorialités investies dans les représentations de ces espaces, même quand les citadins ne perçoivent pas bien, voire n'imaginent pas les continuités végétalisées dans leur ville, incite à se pencher sur l'esthétique environnementale pour donner sens à ces nouveaux espaces qui pourraient participer au développement de ville plus « durables » et favoriser l'investissement citoyen / citadin dans l'appropriation, la co-construction et / ou la cogestion de leur espace de vie. Cette réflexion reviendrait à associer à la préservation de la biodiversité, celle du lien social et du «sens » pour des aménagements dont les services

Développement durable et territoires, Vol. 3, n² 2 | Juillet 2012 
éco-systémiques multiples sont, aujourd'hui, soulignés par les scientifiques comme par les gestionnaires et les politiques.

\section{BIBLIOGRAPHIE}

APUR (Atelier parisien d'urbanisme), 2005, Réaliser de nouveaux jardins, comment végétaliser Paris? Paris, APUR/IDF (http://www.apur.org/sites/default/files/documents/195.pdf)

Berleant A., 1992, The Aesthetics of the environment, Philadelphia, Temple University Press.

Berleant A., 2010, Sensibility and sense. The Aesthetic transfromation of the humain world, St Andrews studies in philosophy and public affairs, Imprint Academic.

Blanc N., 2008, Vers une esthétique environnementale, Versailles, Editions QUAE, Coll. NSS Indisciplines.

Blanc N., Ramos J., 2010, Écoplasties. Art et environnement, Paris, éditions Manuella.

Blanc N., 2012, Nouvelles esthétiques urbaines, Paris, Éditions Armand Colin.

Blanc N., 2008, «Henk A.M.J. Ten Have (Eds), 2007, Éthiques de l'environnement et politique internationale, collection éthiques, Éditions UNESCO, 247 p. », Cybergeo : European Journal of Geography [En ligne], Revue de livres, mis en ligne le 04 juillet 2008, http:// cybergeo.revues.org/19423, consulté le 29 février 2012.

Brady E., 2003, Aesthetics of the natural environment, Edinburgh, Edinburgh University Press.

Brady E., 2007, « Vers une véritable esthétique de l'environnement », in : Lolive J., Blanc N. (dir.), Esthétique et espace public, Paris, Cosmopolitiques, 15, p. 61-73.

Bridge G., 2008, "City senses : on the radical possibilities of pragmatism in geography", Geoforum, $\mathrm{n}^{\circ} 39$, p. 1570-1584.

Carlson A., 1999, Aesthetics and the Environment. The Appreciation of Nature, Art and Architecture, London, Routledge.

Carlson A., Berleant A., 2004, The Aesthetics of natural environments, New York, Broadview Press.

Catterall M. \& Maclaran P., 1997, "Focus group data and qualitative analysis programs", Sociological Research Online, vol. $2, \mathrm{n}^{\circ} 1$.

Communications, 2003, $\mathrm{n}^{\circ}$ 74, « Bienfaisante nature »

Consales J.-M., Goiffon M. et Barthélemy C., 2012, « Entre aménagement du paysage et ménagement de la nature à Marseille : la trame verte à l'épreuve du local », Développement durable et territoires [En ligne], vol. 3, nº 2 | Juillet 2012.

Descola P., 2005, Par-delà nature et culture, Paris, Gallimard.

Foucault M., 2009, Le corps utopique, les hétérotopies, Paris, Nouvelles Editions Lignes.

Dubost F., Lizet B., 2003, « La nature dans la cité », Communications, n 74, p. 5-8.

Gauchet M., 1985, Le Désenchantement du monde. Une histoire politique de la religion, Paris, Gallimard. 
Gobster P. H., Nassauer J. I., Daniel T., Fry G., 2007, “The shared landscape : what does Aesthtics have to do with ecology ?", Landscape ecology, n²2, p. 959-972.

Henk A.M.J, Ten Have (Eds), Éthiques de l'environnement et politique internationale, Paris, Éditions UNESCO, Collection éthiques.

Kalaora B, 1998, Au delà de la nature, l'environnement, Paris, Ed. l'Harmattan,

Lafon P., 1981, « Analyse lexicométrique et recherche des cooccurrences », Mots, $n^{\circ}$ 3. p. 95-148.

Moscovici S., 1972, La société contre nature, Paris, Union générale d'éditions.

Mucchielli R., 2004 (15ème édition), La dynamique des groupes, Paris, ESF Éditions.

Rolston H., 2007, « La terre et ses valeurs intrinsèques : la nature et les nations ", in : Henk A.M.J, Ten Have (Eds), Éthiques de l'environnement et politique internationale, Paris, Éditions UNESCO, Collection éthiques, p. 10-25.

Saito Y., 2010, Everyday Aesthetics, Oxford, Oxford University Press.

\section{RÉSUMÉS}

De quelle trame verte les citadins peuvent-ils rêver? Des focus group ont été organisés dans trois villes françaises ayant des cultures urbaines et environnementales très différentes: Paris, Marseille et Strasbourg. Les débats intervenus entre les citadins réunis nous ont permis de mettre en évidence un rapport à la nature et aux continuités naturelles ainsi que des pratiques notablement différents d'une ville à l'autre. Cependant, les représentations citadines de la nature en ville soulignent l'importance des sensorialités dans tous les cas, ce qui nous incite à investiguer le champ de l'esthétique environnementale, et conduit à une réflexion sur l'appropriation et la participation des citadins à ces aménagements promus par la loi Grenelle II de l'environnement.

What greenway can the city dwellers dream of? Focus groups of city dwellers have been organized in three French cities whith very different urban culture and environmental context : Paris, Marseille and Strasbourg. The debates between the participants allowed us to evidence that what they imagine is part of an environmental esthetic and reveals a relationship with nature which are different from one town to another. Nevertheless, everywhere, urban dwellers' representations of nature and natural continuities reveal the importance of senses. It seems usefull, then, to explore the field of environmental aesthetics that could lead us to think about how citizens could appropriate and participate to these new urban structures promoted by the so called French law "Grenelle II" de l'environnement).

\section{INDEX}

Keywords : greenway, nature, cognitive representations, city, ethics, esthetics, urban practices

Mots-clés : trame verte, nature, représentations cognitives, ville, esthétique, pratiques urbaines 


\section{AUTEURS}

\section{SANDRINE GLATRON}

Sandrine Glatron est géographe. Chargée de recherche au CNRS, ses champs d'investigation sont les représentations cognitives et spatiales qu'ont les citadins des risques urbains et des problèmes environnementaux en ville. CR CNRS, ERL LIVE, 3 rue de l'Argonne, 67000 Strasbourg. Sandrine.glatron@live-cnrs.unistra.fr

\section{ÉTIENNE GRÉSILLON}

Etienne Grésillon est géographe. Maître de conférences à l'Université Paris-Diderot, étudie les liens entre la distribution de la végétation dans les espaces ruraux et urbains et les représentations laïques et religieuses de la nature. MCF Université Paris-Diderot, UMR Ladyss, 105 rue de Tolbiac, 75013 Paris, etienne.gresillon@wanadoo.fr

\section{NATHALIE BLANC}

Nathalie Blanc est géographe. Directrice de recherche au CNRS en géographie (UMR 7533 LADYSS). Ses domaines de recherche concernent le thème de la nature en ville et de l'esthétique environnementale. DR CNRS, UMR Ladyss, Université Paris 7 UFR GHSS Immeuble Montréal, Les Olympiades 105 rue de Tolbiac 75013 Paris, nathali.blanc@wanadoo.fr 